\title{
Population-specific toxicity of six insecticides to the trematode Echinoparyphium sp.
}

\author{
JESSICA HUA ${ }^{1}$, NICHOLAS BUSS ${ }^{2}$, JUSTIN KIM ${ }^{2}$, SARAH A. ORLOFSKE ${ }^{3}$ and \\ JASON T. HOVERMAN ${ }^{2}$ \\ ${ }^{1}$ Biological Sciences Department, Binghamton University (SUNY), Binghamton, New York 13902, USA \\ ${ }^{2}$ Department of Forestry and Natural Resources, Purdue University, West Lafayette, Indiana 47907, USA \\ ${ }^{3}$ Department of Biology, Northeastern Illinois University, Chicago, Illinois 60625, USA
}

(Received 14 September 2015; revised 20 November 2015; accepted 26 November 2015)

\section{SUMMARY}

The ubiquitous use of pesticides has increased concerns over their direct and indirect effects on disease dynamics. While studies examining the effects of pesticides on host-parasite interactions have largely focused on how pesticides influence the host, few studies have considered the effects of pesticides on parasites. We investigated the toxicity of six common insecticides at six environmentally-relevant concentrations to cercariae of the trematode Echinoparyphium from two populations. All six insecticides reduced the survival of cercariae (overall difference between mortality in control $v$ s pesticide exposure $=86 \cdot 2 \pm 8 \cdot 7 \%$ ) but not in a predictable dose-dependent manner. These results suggest that Echinoparyphium are sensitive to a broad range of insecticides commonly used in the USA. The lack of a clear dose-dependent response in Echinoparyphium highlights the potential limitations of toxicity assays in predicting pesticide toxicity to parasites. Finally, population-level variation in cercarial susceptibility to pesticides underscores the importance of accounting for population variation as overlooking this variation can limit our ability to predict toxicity in nature. Collectively, this work demonstrates that consideration of pesticide toxicity to parasites is important to understanding how pesticides ultimately shape disease dynamics in nature.

Key words: Helisoma trivolvis, carbaryl, malathion, cypermethrin, permethrin, imidacloprid, thiamethoxam.

\section{INTRODUCTION}

Infectious diseases are emerging at an unprecedented rate in plant, wildlife and human populations (Daszak et al. 2003; Jones et al. 2008; Tompkins et al. 2015). While a diversity of factors can drive disease dynamics, there is growing awareness that human actions may be a significant contributor. Human activities have dramatically altered the environment through climate change, habitat fragmentation, and introduced species, which can directly or indirectly influence disease dynamics (Blaustein and Kiesecker, 2002; Morley et al. 2003, 2005; Daszak et al. 2003; Jones et al. 2008; Blaustein et al. 2011). Recently, the influence of agrochemicals on disease dynamics has garnered attention. For over 70 years, pesticides have been critical tools for improving human health by enhancing the yield of agricultural systems. However, our increasing reliance on pesticides to control a broad range of pest species has raised concern over whether these chemicals could be playing a role in disease outcomes (Christensen et al. 2006; Gilliom, 2007; Rhind, 2009). For instance, the interaction between parasites and pesticides has been proposed as a contributor to

\footnotetext{
* Corresponding author. Biological Sciences Department, Binghamton University (SUNY), Binghamton, New York 13902, USA. E-mail: jhua@binghamton.edu
}

population declines in amphibians, honeybees and salmon (Blaustein et al. 2011; Dietrich et al. 2014; Doublet et al. 2015). As the human population grows and anthropogenic chemicals continue to contaminate natural systems, consideration of the interactions between pesticides and parasites is fundamental to our understanding of potential factors influencing emerging infectious diseases (Marcogliese and Pietrock, 2011).

Mounting evidence suggests that pesticides can influence disease outcome directly (e.g., mortality) and indirectly (e.g., immune suppression; Carey et al. 1999; Kiesecker, 2002; Christin et al. 2004; Rohr et al. 2008). For instance, Coors and De Meester (2008) found that exposure to the insecticide carbaryl had a synergistic effect on Daphnia magna by increasing susceptibility to the parasite Pasteuria ramosa. Similarly, in amphibians, Rohr et al. (2008) found that green frog tadpoles exposed to the herbicide atrazine had significantly higher levels of encysted trematodes (Echinostoma trivolvis). Further, Kiesecker (2002) linked pesticide exposure to increased infection and pathology (i.e., limb malformations) in amphibians exposed to the trematode Ribeiroia ondatrae. These studies demonstrate that pesticides can influence disease dynamics in complex ways across a wide range of taxa. Investigating pesticide-mediated effects on the transmission phase of host-parasite interactions can 
have broad implications for understanding disease dynamics as well as protecting wildlife populations facing multiple stressors.

While growing evidence suggests that pesticides can influence disease outcomes of hosts (e.g., infection, pathology and mortality), fewer studies have considered how pesticides affect parasites (Koprivnikar et al. 2006; Rohr et al. 2008). Specifically, many parasites (e.g., trematodes) have free-living stages that are likely exposed to pesticide contamination, which can influence parasite survival or behaviour and affect transmission to the host (Koprivnikar et al. 2006). While past evidence suggests that exposure to pesticides during these freeswimming stages may influence parasite mortality, the existing evidence is equivocal. For example, Rohr et al. (2008) exposed E. trivolvis cercariae to two herbicides (atrazine and glyphosate) and two insecticides (malathion and carbaryl) and found that only atrazine $\left(201 \mu \mathrm{g} \mathrm{L}^{-1}\right)$ caused a significant increase in mortality. Similarly, Koprivnikar et al. (2006) found that $200 \mu \mathrm{g} \mathrm{L}^{-1}$ but not $20 \mu \mathrm{g} \mathrm{L} \mathrm{L}^{-1}$ of atrazine caused an increase in mortality relative to the control. Griggs and Belden (2008) found that $100 \mu \mathrm{g} \mathrm{L}^{-1}$ of atrazine did not cause an increase in mortality until it was mixed with $85 \mu \mathrm{g} \mathrm{L}^{-1}$ of another herbicide (metolachlor). Although these studies suggest that pesticides can be toxic to freeliving parasites, our understanding is based on a limited number of pesticides (atrazine, glyphosate, malathion and carbaryl; Koprivnikar et al. 2006; Griggs and Belden, 2008; Rohr et al. 2008). With over 600 different active ingredients currently registered for use in the USA (EPA, 2010a), there is a need to expand our understanding of how parasites respond to a larger diversity of pesticides that vary in mode of action (Newman, 2010; Grube et al. 2011).

Another challenge in pesticide research that may limit our understanding of how pesticides influence disease dynamics is that the toxicity values for different chemicals can show substantial population-level variation. For example, studies across diverse taxa (e.g., water fleas, fairy shrimp, wood frogs and leopard frogs) have found that populations can dramatically differ in their tolerance to pesticides (Bridges and Semlitsch, 2000a; Brausch and Smith, 2009; Cothran et al. 2013; Bendis and Relyea, 2014; Hua et al. 2015). There is also increasing evidence that such population-level variation can be attributed to proximity to agriculture; populations located closer to agricultural fields tend to have higher pesticide tolerance compared with populations farther from agricultural fields (Bridges and Semlitsch, 2000a; Brausch and Smith, 2009; Cothran et al. 2013; Bendis and Relyea, 2014; Hua et al. 2015). Collectively, this research suggests that populations can evolve pesticide tolerance, underscoring the need to examine pesticide toxicity across populations.
However, whether similar population-level differences in pesticide tolerance exist for parasites remains relatively unexplored (but see Morley et al. 2003).

Traditional toxicological approaches rely on the assumption that organisms respond to pesticides in a dose-dependent manner with increasing pesticide levels causing increased mortality (i.e. 'the dose makes the poison'; Calabrese and Baldwin, 2003). However, growing evidence suggests that pesticides and other toxins commonly initiate a hormetic doseresponse curve with lower and higher doses causing higher mortality compared with intermediate doses (Calabrese, 2005). For instance, using heavy metals, Morley et al. (2005) demonstrated that early exposure to lower concentrations of metals actually initiated a protective effect on cercariae of Diplostomum spathaceum. Yet to date, the majority of studies have focused on narrow ranges of concentrations and most often examine the effects of relatively high concentrations of pesticides (i.e., worst-case scenario concentrations; Koprivnikar et al. 2006; Griggs and Belden, 2008; Rohr et al. 2008). For pesticides that initiate hormetic patterns of toxicity, overlooking lethal effects of lower concentrations can obscure our understanding of ecologically relevant concentrations of pesticides on disease dynamics. To increase our ability to predict how pesticides will shape disease dynamics, there is a need for studies that test a broad range of ecologically relevant concentrations to quantify dose-response relationships.

Here, we investigated the toxicity of six common insecticides (carbaryl, malathion, cypermethrin, permethrin, imidacloprid and thiamethoxam) at six environmentally relevant concentrations to two populations of the trematode Echinoparyphium lineage 3 (Detwiler et al. 2010). To date, this study represents the most comprehensive range of concentrations and pesticides, tested on parasites and is the only study that incorporates a range of concentrations large enough to allow for the detection of non-linear responses. Using traditional toxicological experiments, we quantified dose-response curves for parasite survival for each insecticide, compared toxicity across the insecticides, and evaluated the amount of variation in toxicity estimates between the parasite populations.

\section{METHODS}

\section{Study system}

This study focused on the cercarial stage of the trematode Echinoparyphium lineage 3 (Detwiler et al. 2010). The first intermediate host of Echinoparyphium are snails (e.g., Helisoma trivolvis). Echinoparyphium mircacidia penetrate the head foot area of the snail and form into a sporocyst (Kanev et al. 1995). Echinoparyphium then form free-living cercariae, which leave the snails and enter the 
aquatic environment where they infect second intermediate hosts, including both gastropods and amphibian larvae. Once in the second intermediate host, the trematode develops into a metacercaria that encysts in the kidney of the amphibian host or the mantle and pericardium organs in molluscs. Echinoparyphium is widespread across North America and have also been found in Europe, Asia and Africa (Kanev et al. 1995). We chose to focus on the cercarial stage because this free-living stage is most likely in direct contact with pesticides for the longest period of time. Toxic effects of pesticide during this stage may directly influence parasite survival and subsequently transmission to the second intermediate host. Therefore, quantifying these effects on the cercariae stage may help to shed light on how pesticides influence disease dynamics.

\section{Animal collection and husbandry}

On 30 March and 1 April 2015, we collected 25 adult ramshorn snails (H. trivolvis) from two local ponds located in West Lafayette, IN, USA: Purdue Wildlife Area (PWA) and Indian Creek Pond (ICP). PWA $\left(2636 \mathrm{~m}^{2}\right)$ and ICP $\left(3266 \mathrm{~m}^{2}\right)$ are both ponds that are comparable in size and have similar amphibian and snail abundance and diversity (personal observation). PWA is surrounded by forest and ICP is surrounded by agriculture and using Google Earth (2013, v. 7·1·2), we measured the linear distance from each pond centre to the nearest agricultural field using similar methodology described in (Hua et al. 2015). PWA is located 278 $\mathrm{m}$ from agriculture and ICP is located $50 \mathrm{~m}$ from agriculture. Previous research has demonstrated that agricultural practices have the strongest influence on ponds within $150 \mathrm{~m}$ of agricultural land (Declerck et al. 2006). We screened each snail for Echinoparyphium infection by isolating individuals in $50 \mathrm{~mL}$ tubes filled with $45 \mathrm{~mL}$ of aged well water and induced the shedding of the cercariae by placing the tube under a light source for $1 \mathrm{~h}$ (Cohen et al. 1980). We selected three snails from each population that shed the highest density of cercariae and housed them separately in $2 \mathrm{~L}$ plastic containers with $1.5 \mathrm{~L}$ of filtered well water at $7^{\circ} \mathrm{C}$ to slow shedding of cercariae until the start of the experiment. Three days prior to the start of the experiment, snails were slowly acclimated to $25^{\circ} \mathrm{C}$ and fed rabbit chow ad libitum.

\section{Trematode identification}

We identified echinostome cercariae from both ponds through standard molecular sequencing of the ITS1 gene and implementation of Bayesian phylogenetics methods (Supplementary methods in Appendix). Based on our phylogeny (see online Supplementary information; Figure S1), we identified all of our samples as belonging to Echinoparyphium lineage 3 as described by Detwiler et al. (2010).

\section{Experimental design}

Our focal pesticides were six insecticides that vary in mode of action and are commonly used in agricultural and residential settings (Fossen, 2006; Grube et al. 2011; Main et al. 2014). We chose two acetylcholine esterase inhibitors (AChE; carbaryl and malathion), two $\mathrm{Na}^{+}$channel disruptors (cypermethrin and permethrin), and two nicotinic acetylcholine receptor disruptors (nAChr; imidacloprid and thiamethoxam). For each of the two parasite populations, our pesticide treatments were a pesticide-free control and six environmentally relevant concentrations of six different pesticides (see Table A3 for concentrations). These 37 treatments were replicated four times for a total of 296 experimental units $(37$ treatment $\times 4$ replicates $\times 2$ populations). Experimental units were individual wells within a 24 -well plate. To prevent cross contamination of pesticides and to limit the time needed to find and add cercariae to the appropriate experimental unit, we used a separate plate for each insecticide for each population. Thus, for a given population (ICP vs PWA), a single 24-well plate allowed us to test all four replicates of the six concentrations for any given insecticide ( 6 concentrations $\times 4$ replicates/concentration). For the pesticide-free control treatments, we created an additional 24-well plate with four pesticide-free wells for each population. Therefore, we used a total of 14-well plates for the experiment.

\section{Pesticide solutions and testing}

To create the pesticide solutions, we first diluted commercial grade insecticide to make the stock solution for each pesticide. We added $1 \cdot 5 \mu \mathrm{L}$ of the concentrated commercial grade formulation of each insecticide to $1.5 \mathrm{~L}$ of aged filtered water to achieve stock solutions of $0.23,0.5,0.25,0.25,0.24$ and $0 \cdot 24 \mu \mathrm{g} \mu \mathrm{L}^{-1}$ of carbaryl, malathion, cypermethrin, permethrin, imidacloprid and thiamethoxam, respectively. We then used the dilute stock solution to create the concentrations used in the experiment (working solutions). For details regarding the volume of dilute stock solutions added to create each working solution, refer to Appendix (Table A3). We sent samples of the diluted stock solution used to create each of the working solutions to Purdue Bindley Bioscience Center (West Lafayette, IN) for confirmation. For nominal concentrations of $0 \cdot 23,0 \cdot 5,0 \cdot 25,0 \cdot 25,0 \cdot 24$ and $0 \cdot 24 \mu \mathrm{g} \mu \mathrm{L}^{-1}$ of carbaryl, malathion, cypermethrin, permethrin, imidacloprid and thiamethoxam, actual concentrations were $0 \cdot 22,0.4,0 \cdot 3,0.35,0.28$ and $0.28 \mu \mathrm{g} \mu \mathrm{L}^{-1}$, respectively. 


\section{Cercariae addition}

To obtain cercariae for the experiment, we individually placed the six infected snails (three from each population) into $50 \mathrm{~mL}$ Falcon tubes filled with 45 $\mathrm{mL}$ of aged well water and exposed them to a light source for a total of $1 \mathrm{~h}$ to induce the shedding of cercariae. We then mixed together cercariae from the same population. Next, we used a glass pipette to randomly collect ten cercariae for addition to each experimental unit $(N=2960$ total cercariae). To prevent cross-contamination among pesticide concentrations, we added cercariae from the lowest concentration to the highest concentration and switched glass pipettes after every concentration. To keep the age of cercariae as consistent as possible within a plate, we added cercariae to all experimental units within a plate before moving on to the next plate (15-20 min per plate). To control for any potential effect of cercariae age across plates, we randomized the order of plates in which we added cercariae and used newly shed cercariae every $30 \mathrm{~min}$ by moving snails to a new Falcon tube with $45 \mathrm{~mL}$ of fresh water. For the $16 \mu \mathrm{g} \mathrm{L}^{-1}$ treatment of imidacloprid, due to an accidental oversight, we did not add cercariae from PWA to the treated wells.

We terminated the experiment $6 \mathrm{~h}$ after the addition of cercariae to the experimental unit and counted the number of cercariae still alive. A common measure of cercariae survival is the lack of movement after mechanical stimulation (i.e., a stimulus that involves pressure or distortion; Reddy et al. 2004) which can include nudges with a probe or water agitation (Rohr et al. 2008; Griggs and Belden, 2008; Koprivnikar et al. 2006). To assess survival in our experiment, we used a glass pipette to direct a stream of water at the cercariae. Similar to described in Rohr et al. (2008), cercariae were considered dead if they did not move following mechanical stimulation with the directed stream of water. We chose to terminate the experiment at $6 \mathrm{~h}$ because pilot and past studies found that control cercariae mortality outside of a host naturally increases at $6-8 \mathrm{~h}$ and cercariae efficacy is highest within the first $8 \mathrm{~h}$ after shedding (Rohr et al. 2008). In our study, the natural control mortality for cercariae from PWA and ICP at $6 \mathrm{~h}$ was $7 \cdot 5$ and $30 \%$, respectively.

\section{Statistical analysis}

To assess the effects of pesticides on cercariae, we first determined whether pesticides caused an increase in mortality relative to the control. For each of the six pesticides, we ran a separate analysis of variance (ANOVA; SPSS 21) examining the effect of concentration, population and their interaction on cercariae mortality. Using established toxicological protocol, each of the pesticides were compared with a shared control (Newman, 2010). Given that we observed mortality in our control treatments for each population (PWA $-7 \cdot 5 \%$ and ICP $-30 \%$ ), we conducted subsequent analyses that accounted for background mortality to facilitate comparisons between the populations. For these analyses, we transformed mortality using Abbot's formula, a commonly used tool in toxicology (corrected mortality $=$ uncorrected mortality - control mortality/1 - control mortality; Healy, 1952). After correcting for control mortality, we then ran ANOVAs to assess the effects of concentration, population and their interaction on cercariae mortality for each pesticide.

For all analyses, we tested whether the assumptions of ANOVA were satisfied. If the assumptions were violated, we ranked transformed the data for analysis (Quinn and Keough, 2002). For all significant main effects, we conducted Tukey's pairwise comparisons for non-transformed data and Student-NewmanKeuls (SNK) means comparison tests for all ranked data. For significant interactions, we conducted Bonferroni adjusted pairwise comparisons (EMMEANS SPSS 21) to better assess the relationship between the mortality of cercariae at each concentration across populations and also the mortality of cercariae at each concentration within a population. We chose to use ANOVA instead of traditional toxicological analyses (i.e., LC50) because none of the environmentally relevant concentrations we used caused $100 \%$ mortality (a criteria for LC50s).

\section{RESULTS}

\section{AChE inhibitors}

Carbaryl. We found a significant effect on cercariae mortality caused by different carbaryl concentrations $\left(F_{5,36}=17 \cdot 3 ; P<0 \cdot 001\right)$, a marginal effect between the two populations $\left(F_{1,36}=4 \cdot 0 ; P=0.051\right)$, and no significant interaction between these two variables $\left(F_{5,36}=1 \cdot 2 ; P=0 \cdot 33\right)$. All concentrations of carbaryl increased cercariae mortality when compared with the control treatment (Fig. 1; $P<0 \cdot 001$ ).

After accounting for control mortality, we found a significant effect of carbaryl concentration $\left(F_{5,36}=\right.$ $2 \cdot 7 ; P=0 \cdot 04)$, but no effect of population $\left(F_{1,36}=\right.$ $1 \cdot 9 ; P=0 \cdot 17)$ or the interaction $\left(F_{5,36}=0 \cdot 74 ; P=\right.$ $0 \cdot 60)$ on cercariae mortality (Fig. 1). Averaged across populations, $15 \mu \mathrm{g} \mathrm{L}^{-1}$ caused marginally lower mortality compared to $5 \mu \mathrm{g} \mathrm{L}{ }^{-1}(P=0 \cdot 07)$ and $70 \mu \mathrm{g} \mathrm{L}^{-1}(P=0 \cdot 06$; Table S2).

Malathion. We found a significant effect of population $\left(F_{1,36}=26 \cdot 4 ; P<0 \cdot 001\right)$, malathion concentration $\left(F_{5,36}=4 \cdot 6 ; P=0 \cdot 001\right)$, and the interaction $\left(F_{5,36}=7 \cdot 7 ; P<0.001\right)$ on cercariae mortality. In the ICP population, malathion did not influence the mortality of cercariae (Fig. 1; Table A3). 

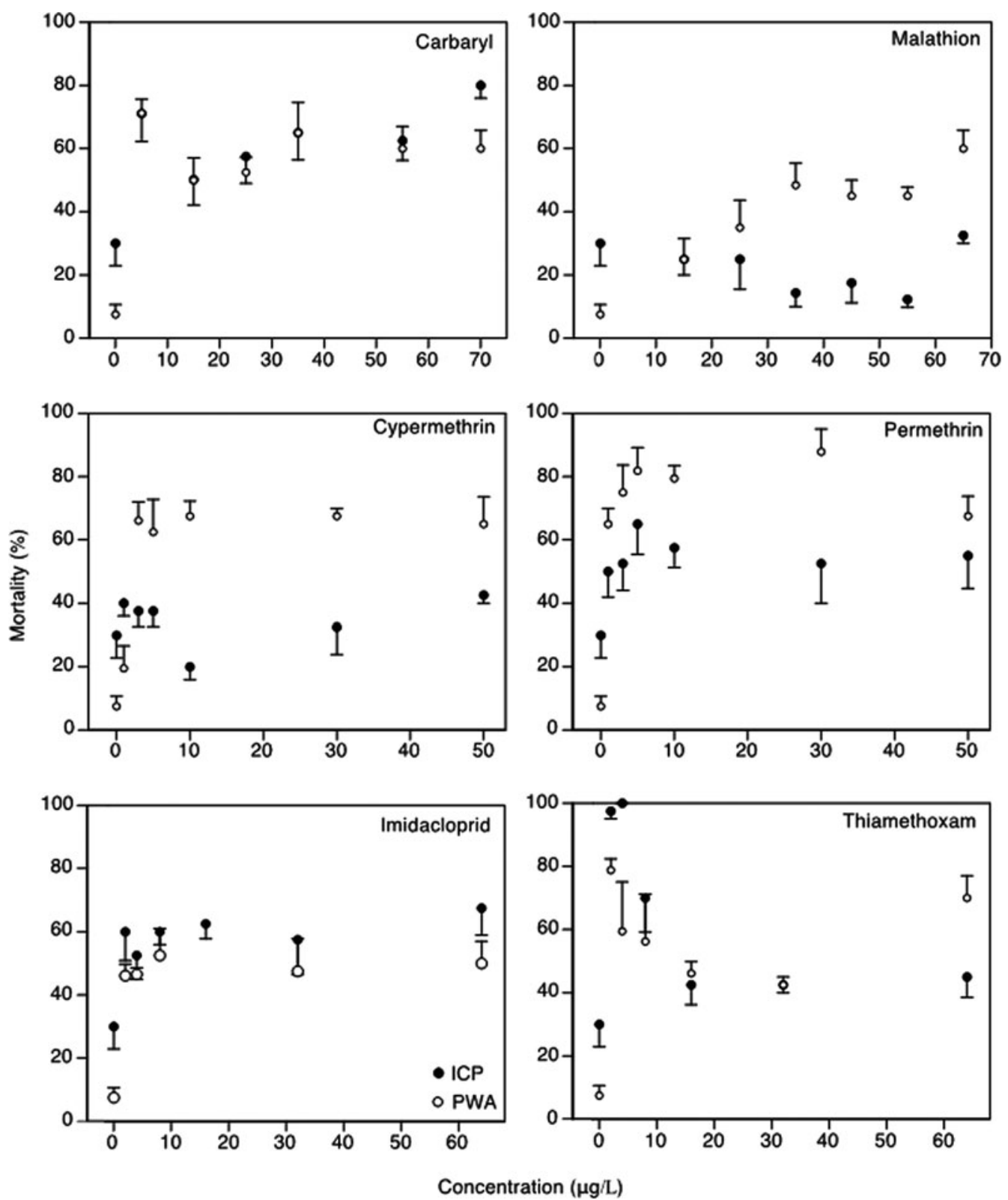

Fig. 1. The mortality (average \pm s.E.) of two populations of Echinoparyphium Lineage 3. cercariae to two acetylcholine esterase inhibiting insecticides (carbaryl and malathion), two $\mathrm{Na}^{+}$channel disruptors (cypermethrin and permethrin), and two nicotinic acetylcholine receptor disruptors (imidacloprid and thiamethoxam). Solid symbols represent the ICP population and open symbols represent the PWA population.

In contrast, for cercariae from PWA, all concentrations of malathion (all $P<0 \cdot 001$ ) except for the lowest concentration $\quad(15 \mu \mathrm{g} \mathrm{L})^{-1} ; \quad P=0 \cdot 367$; Table A3) caused higher mortality relative to the control.

After accounting for control mortality, we found a significant effect of population $\left(F_{1,36}=149 \cdot 7 ; P<\right.$ $0 \cdot 001)$, malathion concentration $\left(F_{5,36}=2 \cdot 8 ; P=\right.$ $0 \cdot 029)$ and the interaction $\left(F_{5,36}=2 \cdot 9 ; P=0 \cdot 02\right)$ on cercariae mortality (Fig. 1). Cercariae from PWA were more susceptible to malathion than those from ICP at all concentrations $(P<0 \cdot 04)$. For cercariae from ICP, mortality was similar across the different malathion concentrations (all $P=1 \cdot 0$ ). In contrast, mortality of cercariae from PWA generally increased with increasing pesticide concentrations. Cercariae exposed to $15 \mu \mathrm{g} \mathrm{L}{ }^{-1}$ had lower mortality compared with 35,45 and $55,65 \mu \mathrm{g} \mathrm{L}^{-1}(P=0 \cdot 02$, $0.07,0.07$ and $P<0.001$, respectively) and cercariae exposed to $25 \mu \mathrm{g} \mathrm{L}{ }^{-1}$ had significantly lower mortality compared with $65 \mu \mathrm{g} \mathrm{L}^{-1}(P=0 \cdot 01)$. All other comparisons were not significant (Table S3).

\section{$\mathrm{Na}^{+}$channel disruptors}

Cypermethrin. We found a significant effect of population $\left(F_{1,36}=27 \cdot 6 ; P<0 \cdot 001\right)$, cypermethrin concentration $\left(F_{5,36}=11.5 ; \quad P<0.001\right)$, and the interaction $\left(F_{5,36}=11 \cdot 5 ; P<0 \cdot 001\right)$ on cercariae mortality. We found no difference in the mortality 
of cercariae from ICP in any of the cypermethrin treatments relative to the control (Fig. 1; Table A3). In contrast, for cercariae from PWA, all cypermethrin concentrations (all $P<0.001$; Table A3) except for the lowest concentration (1 $\left.\mu \mathrm{g} \mathrm{L}^{-1} ; P=0 \cdot 367\right)$ caused higher mortality compared with the control.

After accounting for control mortality, we found a significant effect of population $\left(F_{1,36}=99 \cdot 6 ; P<\right.$ $0 \cdot 001)$, cypermethrin concentration $\left(F_{5,36}=2 \cdot 8 ; P\right.$ $=0.03)$ and the interaction $\left(F_{5,36}=4.7 ; P=0.002\right)$ on cercariae mortality (Fig. 1). At $1 \mu \mathrm{g} \mathrm{L}^{-1}$, there was no difference in mortality between the two populations $(P=1 \cdot 0)$. However, cercariae from PWA were more susceptible to cypermethrin than those from ICP at concentrations $>1 \mu \mathrm{g} \mathrm{L}^{-1}(P \leqslant$ 0.001). For cercariae from ICP, mortality was similar across the different cypermethrin concentrations. In contrast, cercariae from PWA exposed to 1 $\mu \mathrm{g} \mathrm{L}^{-1}$ had significantly lower mortality compared with all other concentrations $(P<0.001$; Table S4).

Permethrin. We found a significant effect of population $\left(F_{1,36}=13 \cdot 1 ; P=0 \cdot 001\right)$, permethrin concentration $\left(F_{5,36}=14.8 ; P<0.001\right)$ and the interaction $\left(F_{5,36}=3 \cdot 4 ; P=0.008\right)$ on cercariae mortality. For ICP, exposure to $5 \mu \mathrm{g} \mathrm{L} \mathrm{L}^{-1}$ of permethrin increased mortality compared with the control. There were no differences in mortality among the other treatments (Fig. 1; Table A3). For PWA, all concentrations of permethrin caused increased mortality compared with the control $(P<0 \cdot 001)$.

After accounting for control mortality, we found a significant effect of population $\left(F_{1,36}=36.9 ; P<\right.$ $0 \cdot 001)$ but no effect of permethrin concentration $\left(F_{5,36}=0.88 ; P=0 \cdot 5\right)$ or the interaction $\left(F_{5,36}=\right.$ $0 \cdot 38 ; P=0.86$ ) on cercariae mortality (Fig. 1). Cercariae from PWA were more susceptible to permethrin compared to cercariae from ICP.

\section{nAChr disruptors}

Imidacloprid. We found a significant effect of population $\left(F_{1,36}=10 \cdot 4 ; P=0.002\right)$ and imidacloprid concentration $\left(F_{5,36}=9 \cdot 2 ; P<0 \cdot 001\right)$, but no interaction $\left(F_{5,36}=0 \cdot 46 ; P=0 \cdot 81\right)$ on cercariae mortality. In both populations, all concentrations of imidacloprid caused higher cercariae mortality relative to the control $(P \leqslant 0 \cdot 001)$. After accounting for control mortality, we found no effect of population $\left(F_{1,36}=0.13 ; P=0.72\right)$, imidacloprid concentration $\left(F_{5,36}=0.41 ; P=0 \cdot 84\right)$, or the interaction $\left(F_{5,36}=\right.$ $0 \cdot 24 ; P=0 \cdot 91$ ) on cercariae mortality (Fig 1 ).

Thiamethoxam. We found a marginally significant effect of population $\left(F_{1,36}=2.9 ; P=0.09\right)$, a significant effect of thiamethoxam concentration $\left(F_{5,36}=\right.$ $22 \cdot 1 ; P<0 \cdot 001)$, and a significant interaction $\left(F_{5,36}\right.$ $=3.8 ; P=0.004)$ on cercariae mortality. Cercariae from ICP exposed to $2 \mu \mathrm{g} \mathrm{L}^{-1}(P<0 \cdot 001), 4 \mu \mathrm{g} \mathrm{L}^{-1}$ $(P<0.001)$ and $8 \mu \mathrm{g} \mathrm{L}^{-1}(P=0.003)$ of thiamethoxam had higher mortality compared with the control treatment (Fig. 1). In contrast, for cercariae from PWA, all concentrations of thiamethoxam $(P<0.001)$ caused higher mortality relative to the control.

Finally, after accounting for control mortality, we found a significant effect of population $\left(F_{1,36}=6 \cdot 2\right.$; $P=0.02)$ and thiamethoxam concentration $\left(F_{5,36}=\right.$ 8.1; $P<0.001$ ) on cercariae mortality (Fig. 1 ), but no interaction $\left(F_{5,36}=2 \cdot 2 ; P=0 \cdot 08\right)$. Cercariae from ICP were more tolerant of thiamethoxam compared to cercariae from PWA. Cercariae exposed to $2 \mu \mathrm{g}$ $\mathrm{L}^{-1}$ had significantly higher mortality compared to all other concentrations $(P<0 \cdot 04)$ except for cercariae exposed to 4 and $8 \mu \mathrm{g} \mathrm{L}^{-1}$ and cercariae exposed to 4 $\mu \mathrm{g} \mathrm{L}^{-1}$ had significantly higher mortality compared with $16(P=0 \cdot 01)$ and $32 \mu \mathrm{g} \mathrm{L}^{-1}(P=0 \cdot 004)$.

\section{DISCUSSION}

As wildlife diseases emerge at unprecedented rates, understanding the contribution of factors such as pesticides to disease dynamics has broad implications. While the majority of studies have examined the influence of pesticides on host susceptibility and pathology, fewer studies have assessed the effects of pesticides on parasites, especially those with free-living stages (Rohr et al. 2008). In this study, we investigated the toxicity of six common insecticides at six environmentally relevant concentrations on two populations of Echinoparyphium cercariae. At ecologically relevant concentrations, we found that these pesticides reduced the survival of cercariae but not in the expected linear dose-dependent manner. Moreover, we found that cercariae mortality was highly population and pesticide specific.

We examined the toxicity of six insecticides representing three chemical classes to the cercariae of Echinoparyphium. We discovered that all of the insecticides increased the mortality of cercariae. Despite the variation between the populations in the amount of mortality, these results suggest that Echinoparyphium are sensitive to a broad range of insecticides that are commonly used in the USA (Gilliom, 2007; Stone et al. 2014). While several studies have examined the effects of herbicides on cercariae (Koprivnikar et al. 2006; Griggs and Belden, 2008; Rohr et al. 2008), few have considered insecticides and none have examined the effects on Echinoparyphium. Rohr et al. (2008) found that $9 \cdot 6 \mu \mathrm{g} \mathrm{L}^{-1}$ of malathion and $33 \cdot 5 \mu \mathrm{g} \mathrm{L} \mathrm{L}^{-1}$ of carbaryl did not increase mortality of $E$. trivolvis cercariae. However, for Echinoparyphium, carbaryl and malathion both caused mortality across a broad range of concentrations in one or both of our populations. We also found that cypermethrin, permethrin, imidacloprid and thiamethoxam were toxic to cercariae, which has not been previously reported for these 
pesticides. According to the EPA categories of toxicity for aquatic organisms (EPA, 2010b), pesticide with an LC50 value $<0 \cdot 1 \mathrm{mg} \mathrm{L}^{-1}$ are considered 'very highly toxic.' While we were unable to calculate LC50 because our highest concentration did not cause $100 \%$ mortality, it is likely that cercariae would fall within this 'very highly toxic' category as concentrations we used were below $<0 \cdot 1 \mathrm{mg} \mathrm{L}^{-1}$ and many caused $>50 \%$ mortality. Therefore, our results suggest that insecticides at a broad range of ecologically relevant concentrations are generally toxic to Echinoparyphium cercariae.

There was also substantial variation between the two cercariae populations in the toxicity of the pesticides. Overall, Echinoparyphium cercariae from ICP were more tolerant relative to cercariae from PWA. A growing body of research has found that tolerance is related to distance to agriculture with populations closer to agriculture having higher pesticide tolerance (Brausch and Smith, 2009; Coors et al. 2009; Cothran et al. 2013; Bendis and Relyea, 2014; Hua et al. 2015). Similarly, we found that the more pesticide tolerant population (ICP) was closer to agriculture $(50 \mathrm{~m})$ relative to the less tolerant population (PWA, $278 \mathrm{~m}$ ). While our study is the first to document population-level variation in cercarial susceptibility to pesticides, this pattern is consistent with previous work in amphibians and zooplankton (Cothran et al. 2013; Bendis and Relyea, 2014; Hua et al. 2015). This study is consistent with the prediction that distance to agriculture plays an important role in driving population responses to pesticides, but future studies that consider these patterns across more populations are necessary. As toxicity may depend on the evolutionary history of the target population, overlooking population-level variation in susceptibility to pesticides can limit our ability to predict toxicity in nature and to make generalizations about pesticide toxicity.

Toxicological theory typically assumes dose-dependent responses such that mortality increases with increasing toxin concentration (Newman, 2010). Surprisingly, for Echinoparyphium, we found that cercarial mortality rarely occurred in the expected linear dose-dependent manner. The only insecticide that initiated a linear dose-dependent response curve was malathion. In contrast, for the majority of the other insecticides, mortality typically plateaued as concentrations increased. The plateau response suggests that there are a subset of individuals that are tolerant of a broad range of concentrations and future work that explores a wider range of concentrations are necessary to determine whether this tolerance is generalizable.

While we did not detect a classic dose-dependent response, two insecticides initiated a hormetic-like dose response. Hormesis is a response in which exposure to an intermediate concentration of a chemical agent is less severe relative to lower or higher concentrations (Hayes et al. 2002; Mattson, 2008). Indeed, for both populations of Echinoparyphium, we detected a hormetic-like curve when cercariae were exposed to carbaryl and thiamethoxam. An increasing number of studies have reported hormetic responses to environmental toxins challenging the traditional dose-dependent paradigm used in environmental toxicology (Calabrese and Baldwin, 2003; Morley et al. 2003; Calabrese, 2005; Costantini et al. 2010). This is the first evidence of hormesis in trematode responses to pesticides and this discovery corroborates the evidence suggesting that hormesis is a common phenomenon across a diversity of taxa. Thus, the growing evidence for hormetic-dose responses highlights the value of considering the potential for hormesis (i.e., incorporating and assessing mortality at both low and high doses) in future studies of toxicology (Calabrese, 2005). Although traditional linear dose-dependent responses were not common in our data, it was clear that exposure to environmentally relevant pesticide concentrations increased cercariae mortality and the magnitude of this increase was dependent on insecticide type.

In conclusion, we demonstrated that Echinoparyphium are directly susceptible to a diversity of commonly used pesticides. However, Echinoparyphium rarely respond to pesticides in the expected linear dose-dependent manner and responses were highly pesticide and population specific. Collectively, we conclude that pesticides can potentially influence disease dynamics by causing direct mortality to the cercariae stage of parasites. In addition to considering the direct effects of pesticides on cercariae survival, future areas of research should consider: (1) how pesticides influence infection success, i.e. the number of metacercariae in the host, (2) more parasite populations and (3) additional parasite species. Further, to begin developing generalizations about how pesticides may directly influence cercariae and ultimately how they may shape disease dynamics in nature, future studies should also consider a broader range of pesticide concentrations and working with insecticides cocktails to explore additive, synergistic or antagonistic effects. Finally, pesticides can have direct and indirect effects on each player in the host-parasite interaction. However, we did not consider the effects of these pesticides on intermediate hosts. As suggested by past studies, pesticides may also cause an increase in host susceptibility (Kiesecker, 2002; Rohr et al. 2008). Therefore, despite increased cercariae mortality, pesticide may still have no net influence on disease outcomes (e.g., infection and pathology). Moreover, a number of studies have demonstrate that hosts can vary in susceptibility to both pesticides and parasites (Bridges and Semlitsch, 2000b; Searle et al. 2011; Cothran et al. 2013; Hua and Relyea, 2014; Bradley et al. 2015). Because pesticides can have direct and indirect effects on hosts and parasites, future studies 
that continue to tease apart the complex effects of pesticides on the different components of the host-parasite interaction are necessary (Rohr et al. 2008).

\section{SUPPLEMENTARY MATERIAL}

To view supplementary material for this article, please visit http://dx.doi.org/10.1017/S0031182015001894

\section{ACKNOWLEDGEMENTS}

We thank Sam Guffey and George Meindl for their help in collecting the snails for this experiment.

\section{FINANCIAL SUPPORT}

This work was funded by a Purdue Postdoctoral Scholars in Natural Resources fellowship to J.H., a Purdue University College of Agriculture AgSEED award to J. T.H., and a Committee for Organized Research Grant from Northeastern Illinois University to S.A.O.

\section{REFERENCES}

Bendis, R. J. and Relyea, R. A. (2014). Living on the edge: populations of two zooplankton species living closer to agricultural fields are more resistant to a common insecticide. Environmental Toxicology and Chemistry/ SETAC 33, 2835-2841.

Blaustein, A. R. and Kiesecker, J. M. (2002). Complexity in conservation: lessons from the global decline of amphibian populations. Ecology Letters 5, 597-608.

Blaustein, A. R., Han, B. A., Relyea, R. A., Johnson, P. T. J., Buck, J. C., Gervasi, S. S. and Kats, L. B. (2011). The complexity of amphibian population declines: understanding the role of cofactors in driving amphibian losses. Annals of the New York Academy of Sciences 1223, 108-119.

Bradley, P. W., Gervasi, S. S., Hua, J., Cothran, R. D., Relyea, R. A., Olson, D. H. and Blaustein, A. R. (2015). Differences in sensitivity to the fungal pathogen Batrachochytrium dendrobatidis among amphibian populations. Conservation Biology 29, 1347-1356.

Brausch, J. and Smith, P. N. (2009). Mechanisms of resistance and crossresistance to agrochemicals in the fairy shrimp Thamnocephalus platyurus (Crustacea: Anostraca). Aquatic Toxicology 92, 140-145.

Bridges, C. M. and Semlitsch, R.D. (2000a). Variation in Pesticide Tolerance of Tadpoles among and within Species of Ranidae and Patterns of Amphibian decline. Conservation Biology 14, 1490-1499. Bridges, C. M. and Semlitsch, R. D. (2000b). Variation in pesticide tolerance of tadpoles among and within species of Ranidae and patterns of amphibian decline. Conservation Biology 14, 1490-1499.

Calabrese, E. J. (2005). Paradigm lost, paradigm found: the re-emergence of hormesis as a fundamental dose response model in the toxicological sciences. Environmental Pollution 138, 378-411.

Calabrese, E. J. and Baldwin, L. A. (2003). Hormesis: the dose-response revolution. Annual Review of Pharmacology and Toxicology 43, 175-197.

Carey, C., Cohen, N. and Rollins-Smith, L. (1999). Amphibian declines: an immunological perspective. Developmental $\sigma^{\circ}$ Comparative Immunology 23, 459-472.

Christensen, M. R., Graham, M. D., Vinebrooke, R. D., Findlay, D. L., Patterson, M. J. and Turner, M. A. (2006). Multiple anthropogenic stressors cause ecological surprises in boreal lakes. Global Change Biology 12, 2316-2322.

Christin, M.S., Ménard, L., Gendron, A.D., Ruby, S., Cyr, D., Marcogliese, D. J., Rollins-Smith, L. and Fournier, M. (2004). Effects of agricultural pesticides on the immune system of Xenopus laevis and Rana pipiens. Aquatic Toxicology 67, 33-43.

Cohen, L. M., Neimark, H. and Eveland, L. K (1980). Schistosoma mansoni: response of cercariae to a thermal gradient. The fournal of Parasitology 66, 362-364

Coors, A. and De Meester, L. (2008). Synergistic, antagonistic and additive effects of multiple stressors: predation threat, parasitism and pesticide exposure in Daphnia magna. Fournal of Applied Ecology 45, 1820-1828.

Coors, A., Vanoverbeke, J., De Bie, T. and De Meester, L. (2009). Land use, genetic diversity and toxicant tolerance in natural populations of Daphnia magna. Aquatic Toxicology 95, 71-79.
Costantini, D., Metcalfe, N. B. and Monaghan, P. (2010). Ecological processes in a hormetic framework. Ecology Letters 13, 1435-1447.

Cothran, R. D., Brown, J. M. and Relyea, R.A. (2013). Proximity to agriculture is correlated with pesticide tolerance: evidence for the evolution of amphibian resistance to modern pesticides. Evolutionary Applications 6, 832-841.

Daszak, P., Cunningham, A. A. and Hyatt, A. D. (2003). Infectious disease and amphibian population declines. Diversity and Distributions 9, 141-150.

Declerck, S., De Bie, T., Ercken, D., Hampel, H., Schrijvers, S., Van Wichelen, J., Gillard, V., Mandiki, R., Losson, B., Bauwens, D., Keijers, S., Vyverman, W., Goddeeris, B., De Meester, L., Brendonck, L. and Martens, K. (2006). Ecological characteristics of small farmland ponds: associations with land use practices at multiple spatial scales. Biological Conservation 131, 523-532.

Detwiler, J. T., Bos, D. H. and Minchella, D. J. (2010). Revealing the secret lives of cryptic species: Examining the phylogenetic relationships of echinostome parasites in North America. Molecular Phylogenetics and Evolution 55, 611-620.

Dietrich, J. P., Van Gaest, A. L., Strickland, S. A. and Arkoosh, M. R. (2014). The impact of temperature stress and pesticide exposure on mortality and disease susceptibility of endangered Pacific salmon. Chemosphere 108, 353-359.

Doublet, V., Labarussias, M., de Miranda, J. R., Moritz, R. F. A. and Paxton, R. J. (2015). Bees under stress: sublethal doses of a neonicotinoid pesticide and pathogens interact to elevate honey bee mortality across the life cycle. Environmental Microbiology 17, 969-983.

EPA $(2010 a)$. Chemical Safety and Pollution Prevention.

EPA (2010b). Appendix I. Toxicity Categories and LOCs.

Fossen, M. (2006). Environmental Fate of Imidacloprid. Department of Pesticide Regulation, Sacramento, CA.

Gilliom, R. J. (2007). Pesticides in U.S. streams and groundwater. Environmental Science and Technology 41, 3408-3414.

Griggs, J. L. and Belden, L. K. (2008). Effects of atrazine and metolachlor on the survivorship and infectivity of Echinostoma trivolvis trematode cercariae. Archives of Environmental Contamination and Toxicology 54, 195-202. Grube, A., Donaldson, D., Kiely, T. and Wu, L. (2011). Pesticides Industry Sales and usage: 2006 and 2007 Market Estimates. U.S. EPA, Washington, DC.

Hayes, T. B., Collins, A., Lee, M., Mendoza, M., Noriega, N., Stuart, A. A. and Vonk, A. (2002). Hermaphroditic, demasculinized frogs after exposure to the herbicide atrazine at low ecologically relevant doses. Proceedings of the National Academy of Sciences of the United States of America 99, 5476-5480.

Healy, M. J. R. (1952). A table of Abbott's correction for natural mortality. Annals of Applied Biology 39, 211-212.

Hua, J. and Relyea, R. (2014). Chemical cocktails in aquatic systems: pesticide effects on the response and recovery of $>20$ animal taxa. Environmental Pollution 189, 18-26.

Hua, J., Jones, D. K., Mattes, B. M., Cothran, R. D., Relyea, R. A. and Hoverman, J. T. (2015). The contribution of phenotypic plasticity to the evolution of insecticide tolerance in amphibian populations. Evolutionary Applications 8, 586-596.

Jones, K. E., Patel, N. G., Levy, M. A., Storeygard, A., Balk, D., Gittleman, J. L. and Daszak, P. (2008). Global trends in emerging infectious diseases. Nature 451, 990-993.

Kanev, I., Fried, B., Dimitrov, V. and Radev, V. (1995). Redescription of Echinostoma trivolvis (Cort, 1914) (Trematoda: Echinostomatidae) with a discussion on its identity. Systematic Parasitology 32, 61-70.

Kiesecker, J. M. (2002). Synergism between trematode infection and pesticide exposure: a link to amphibian limb deformities in nature? Proceedings of the National Academy of Sciences of the United States of America 99, 9900-9904.

Koprivnikar, J., Forbes, M. R. and Baker, R. L. (2006). Effects of atrazine on cercarial longevity, activity, and infectivity. Fournal of Parasitology 92, 306-311.

Main, A. R., Headley, J. V., Peru, K. M., Michel, N. L., Cessna, A. J. and Morrissey, C. A. (2014). Widespread use and frequent detection of Neonicotinoid insecticides in wetlands of Canada's Prairie Pothole region. PLoS ONE 9, e92821.

Marcogliese, D. J. and Pietrock, M. (2011). Combined effects of parasites and contaminants on animal health: parasites do matter. Trends in Parasitology 27, 123-130.

Mattson, M. P. (2008). Hormesis defined. Ageing Research Reviews 7, 1-7. Morley, N. J., Irwin, S. W. B. and Lewis, J. W. (2003). Pollution toxicity to the transmission of larval digeneans through their molluscan hosts. Parasitology 126, S5-S26.

Morley, N. J., Crane, M. and Lewis, J. W. (2005). Toxicity of cadmium and zinc mixtures to cercarial tail loss in Diplostomum spathaceum 
(Trematoda:Diplostomidae). Ecotoxicology and Environmental Safety 60, 53-60.

Newman, M. C. (2010). Fundamentals of Ecotoxicology. CRC Press, Boca Raton, FL.

Quinn, G.P. and Keough, M. J. (2002). Experimental Design and Data Analysis for Biologists. Cambridge University Press, New York, NY.

Reddy, A., Ponder, E. L. and Fried, B. (2004). Effects of copper sulfate toxicity on cercariae and metacercariae of Echinostoma caproni and Echinostoma trivolvis and on the survival of Biomphalaria glabrata snails. Fournal of Parasitology 90, 1332-1337.

Rhind, S. M. (2009). Anthropogenic pollutants: a threat to ecosystem sustainability? Philosophical Transactions of the Royal Society B: Biological Sciences 364, 3391-3401.
Rohr, J.R., Raffel, T.R., Sessions, S. K. and Hudson, P. J. (2008). Understanding the net effects of pesticides on amphibian trematode infections. Ecological Applications 18, 1743-1753.

Searle, C. L., Gervasi, S. S., Hua, J., Hammond, J. I., Relyea, R. A., Olson, D. H. and Blaustein, A. R. (2011). Differential host susceptibility to Batrachochytrium dendrobatidis, an emerging amphibian pathogen. Conservation Biology: The Fournal of the Society for Conservation Biology 25, 965-974.

Stone, W. W., Gilliom, R. J. and Ryberg, K. R. (2014). Pesticides in U. S. streams and rivers: occurrence and trends during 1992-2011 Environmental Science \& Technology 48, 11025-11030.

Tompkins, D. M., Carver, S., Jones, M. E., Krkošek, M. and Skerratt, L.F. (2015). Emerging infectious diseases of wildlife: a critical perspective. Trends in Parasitology 31, 149-159. 